\title{
Design de Interação no Desenvolvimento Distribuído de Software
}

\author{
Daniel Domingos Alves \\ Universidade Federal da Bahia (UFBA) \\ Salvador, Brasil \\ daniel.domingos@ufba.br
}

\author{
Ecivaldo de Souza Matos \\ Universidade Federal da Bahia (UFBA) \\ Salvador, Brasil \\ ecivaldo@ufba.br
}

\begin{abstract}
A maioria das soluções (abordagens, métodos, técnicas) de design de interação é concebida para ser utilizada em ambientes com participantes colocalizados. No entanto, é crescente o número de projetos de software que são desenvolvidos em ambientes com participantes distribuídos geograficamente. $\mathrm{O}$ avanço em desenvolvimento distribuído de software (DDS), aumento no número de projetos e usuários de free/libre open source software (FLOSS), problemas relacionados à interação humano-computador em DDS e desafios de design de interação em DDS motivam a condução deste estudo. Este artigo apresenta nossos esforços atuais para conceber um modelo de processo de design de interação para DDS. Como resultados parciais, identificou-se desafios, problemas e soluções de design de interação em DDS por meio de um mapeamento sistemático. Utilizando informações coletadas e analisadas por meio desse mapeamento, concebeu-se um modelo baseado em evidências para melhoria de design de interação em DDS, a ser avaliado nas próximas etapas da pesquisa.
\end{abstract}

\section{ACM Classification Keywords}

H.5.m. Information Interfaces and Presentation (e.g. HCI): Miscellaneous; D.2.m Software Engineering: Miscellaneous

\section{Author Keywords}

Interação Humano-Computador, Design de Interação, Desenvolvimento Distribuído de Software, Free/Libre Open Source Software

\section{INTRODUÇÃO}

O número de projetos de software e estudos relacionados ao desenvolvimento distribuído de software (DDS) ${ }^{1}$ e free/libre

\footnotetext{
${ }^{1} \mathrm{O}$ termo "DDS", no contexto deste estudo, está relacionado ao desenvolvimento de software com stakeholders distribuídos geograficamente.
}

Permission to make digital or hard copies of all or part of this work for personal or classroom use is granted without fee provided that copies are not made or distributed for profit or commercial advantage and that copies bear this notice and the full citation on the first page. Copyrights for components of this work owned by others than the author(s) must be honored. Abstracting with credit is permitted. To copy otherwise, or republish, to post on servers or to redistribute to lists, requires prior specific permission and/or a fee. Copyright 2018 SBC.

IHC 2018, Anais Estendidos do XVII Simpósio Brasileiro sobre

Fatores Humanos em Sistemas Computacionais

Outubro 22-26, 2018, Belém, Brasil

WTD-IHC open source software (FLOSS) $)^{2}$ tem aumentado nos últimos anos [8, 21, 15]. Projetos de desenvolvimento de software estão cada vez mais sendo conduzidos por participantes distribuídos geograficamente [21, 8, 12]. Empresas de ambientes de desenvolvimento colaborativo (SourceForge ${ }^{3}$, GitHub ${ }^{4}$ ) reportam a sua expansão. Atualmente, há mais de 3,7 milhões de usuários e 430.000 projetos de software registrados no SourceForge [25]. Em 2017, a comunidade do GitHub atingiu 24 milhões de desenvolvedores trabalhando em 67 milhões de repositórios [10]. Somado a isso, a adoção de FLOSS tem crescido na iniciativa privada, em órgão públicos e por usuários finais $[21,8,26]$.

Apesar dos avanços em DDS, a literatura científica evidencia vários problemas relacionados à interação humanocomputador em DDS. Há problemas relacionados à usabilidade [7, 18], à acessibilidade [1] e à experiência de usuário [27, 4]. Há desafios em envolver usuários finais no processo de desenvolvimento de software $[19,5]$ e falta de especialistas em Interação Humano-Computador (IHC) e/ou dificuldades em envolvê-los [5, 14].

Embora vários estudos tenham feito avanços significativos na identificação de desafios e propostas de novas soluções em/para DDS [16, 15], do ponto de vista de Engenharia de Software, DDS não tem sido amplamente abordado pela literatura científica de IHC [7, 14, 2].

O processo de design de interação no contexto distribuído apresenta algumas características que o torna diferente do processo de design de interação em que os participantes estão colocalizados, por exemplo: partes interessadas distribuídas em várias dimensões de tempo, espaço e/ou organização [11]; necessidade de sistemas colaborativos para a realização das atividades; diferentes culturas e hábitos organizacionais. Diante disso, soluções de design de interação, geralmente concebidas para o contexto em que os participantes estão colocalizados, podem não ser adequadas para o contexto distribuído.

Considerando esse contexto e baseado em resultados parciais desta pesquisa [2] e estudos anteriores [19, 5, 28], verificou-se gaps de pesquisas sobre a integração de design de interação com DDS, novas formas de envolvimento de usuários em projetos de DDS e, principalmente, soluções para problemas e

\footnotetext{
${ }^{2}$ Geralmente, em projetos e comunidades de FLOSS, os stakeholders estão distribuídos geograficamente.

${ }^{3}$ https://sourceforge.net/

${ }^{4}$ https://github.com/
} 
desafios atuais de design de interação em DDS. Essa situação demanda novas soluções (modelos, métodos, técnicas, ferramentas) de design de interação e/ou adaptações de soluções existentes para configurações distribuídas.

Diante do exposto, problematiza-se a seguinte questão norteadora de pesquisa:

Qual seria um modelo de design de interação adequado às necessidades das equipes de desenvolvimento distribuído de software?

Este artigo descreve os esforços atuais para conceber um modelo de processo de design de interação para DDS. Como resultados parciais, identificou-se desafios, problemas e soluções de design de interação em DDS por meio de um mapeamento sistemático. Utilizando informações coletadas e analisadas por meio desse mapeamento, concebeu-se um modelo baseado em evidências para melhoria de design de interação em DDS.

Nas próximas seções serão apresentados alguns estudos que abordam design de interação em DDS, o percurso metodológico da pesquisa, os resultados parciais, as contribuições esperadas e as considerações parciais.

\section{DESIGN DE INTERAÇÃO EM DDS}

Esta seção apresenta um breve background sobre estudos que têm sido conduzidos envolvendo design de interação e DDS.

Design de interação tem se consolidado como uma prática com potencial de apoiar projetos de sistemas interativos em direção à melhoria da qualidade do produto em relação a vários aspectos da interação humano-computador, como envolver usuários no processo de desenvolvimento de software; reduzir aspectos negativos da experiência de usuário; desenvolver produtos interativos acessíveis, fáceis e eficientes [20].

Audy e Prikladnicki [3, p. 54] afirmam que "O desenvolvimento distribuído de software caracteriza-se pela distância física e/ou temporal entre alguns elementos (cliente, usuário e desenvolvedores, por exemplo) envolvidos no processo de desenvolvimento". Segundo Carmel [6], as principais características que diferenciam DDS do desenvolvimento de software tradicional (colocalizado) são distância, diferença de fuso horário e diferenças culturais.

Um dos contextos mais triviais de desenvolvimento distribuído de software é o de projetos de FLOSS. O desenvolvimento de FLOSS normalmente é realizado por equipes auto-organizadas e distribuídas geograficamente e baseia-se em contribuições voluntárias através da Internet [23, 22].

Alguns estudos [19, 5] e resultados preliminares desta pesquisa [2] têm destacado o gap entre design de interação e DDS. Esses estudos destacam alguns problemas e desafios, por exemplo, a falta de integração de design de interação e DDS, desafios no envolvimento de usuários e especialistas em IHC e a qualidade do software desenvolvido.

Algumas iniciativas e propostas têm envolvido design de interação e DDS. Há também estudos que relatam a condução de design de interação com equipes distribuídas geograficamente. Alguns desses estudos são apresentados a seguir.
Lichtner et al. [17] reportam um estudo de caso sobre o uso de diário de usuário online para a avaliação remota do protótipo de um software. A avaliação foi realizada de forma colaborativa em quatro organizações, em diferentes países europeus. Os autores afirmam que o diário possibilitou a comunicação entre usuários, pesquisadores e desenvolvedores do sistema, dando voz aos usuários no processo de avaliação e redesign do software.

Luz e Masoodian [19] descrevem o processo de design de um sistema interativo realizado em comunidades remotas na região amazônica. Os autores afirmam ter aplicado uma abordagem de design participativo para envolver usuários, designers e desenvolvedores distribuídos geograficamente.

Walsh e Foss [28] descrevem o processo de design de um ambiente online para suportar codesign distribuído e intergeracional. Os autores criaram um ambiente de design online baseado na Web, denominado Online Kidsteam.

\section{PERCURSO METODOLÓGICO}

O percurso metodológico desta pesquisa constitui-se de três fases: exploratória; empírica; e desenvolvimento e avaliação da solução, as quais são descritas a seguir.

Fase I - Exploratória: constituiu-se de uma pesquisa bibliográfica por meio de revisão de literatura $a d$ hoc e dois mapeamentos sistemáticos para entender o tema relacionado à pesquisa e identificar problemas, desafios e soluções de design de interação em DDS.

Fase II - Empírica: envolve um estudo empírico em projetos de DDS por meio de netnografia e a pesquisa survey. Essa fase visa nos auxiliar na compreensão do processo de design de interação em ambientes de DDS e identificar soluções de design de interação em projetos de software com participantes distribuídos geograficamente. Essa fase será dividida em duas etapas: (i) coleta de dados por meio de questionários e netnografia e (ii) análise dos dados.

Fase III - Desenvolvimento e avaliação da solução: refere-se ao desenvolvimento e à avaliação de artefatos para apoiar a solução de problemas e desafios de design de interação em DDS. Pretende-se conceber dois artefatos: (i) modelo baseado em evidências para melhoria de design de interação em DDS e (ii) modelo de processo de design de interação para DDS. O primeiro modelo foi concebido a partir dos resultados da fase I e orientará as atividades para a concepção e avaliação do segundo modelo. Os resultados das fases I e II serão utilizados para conceber o modelo de processo de design de interação para DDS. O modelo de processo de design de interação para DDS será avaliado por especialistas e aplicado a um ou mais projetos de DDS.

A metodologia desta pesquisa foi elaborada com base no método proposto por Dresch, Lacerda e Antunes [9] para a condução da design science research, nas diretrizes de pesquisa em design science propostas por Hevner et al. [13], na metodêutica [24] e considerando os objetivos desta pesquisa. Para atingi-los, os passos metodológicos ilustrados na Figura 1 estão sendo seguidos. 


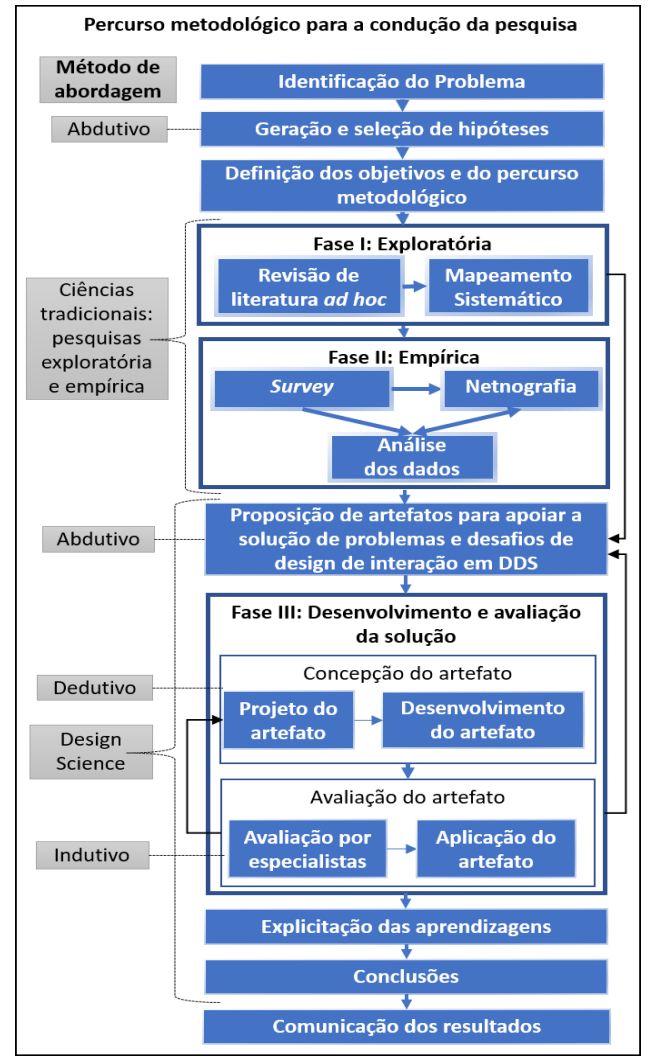

Figure 1. Percurso metodológico da pesquisa. Modificado de [9].

\section{RESULTADOS PARCIAIS E CONTRIBUIÇÕES}

Um mapeamento sistemático foi conduzido para identificar estudos que relatam sobre métodos, técnicas, ferramentas, abordagens e estratégias de design de interação propostos/utilizados para/no desenvolvimento de FLOSS [2]. Os resultados desse mapeamento mostraram que há poucos estudos que envolvem design de interação e desenvolvimento de FLOSS. Devido ao baixo número de estudos encontrados nesse mapeamento, optou-se por ampliar o escopo desta pesquisa, que passou de design de interação em desenvolvimento de FLOSS para design de interação em DDS.

Uma vez que o escopo da pesquisa foi ampliado, um novo mapeamento sistemático se fez necessário. Desta vez para identificar e sintetizar estudos que descrevem desafios, problemas e soluções de design de interação em DDS. Nesse novo mapeamento, foram identificados muitos problemas e desafios e poucas soluções de design de interação em DDS.

Utilizando os resultados do mapeamento sistemático, concebeu-se um modelo para melhoria de design de interação em DDS. O modelo visa orientar pesquisadores na condução de pesquisas para melhoria de design de interação em DDS.

Na próxima etapa será conduzido um estudo empírico, com o objetivo de verificar como tem sido conduzido o processo de design de interação em DDS e identificar soluções de design de interação utilizadas em projetos de software com participantes distribuídos geograficamente. Com isso, espera-se propiciar fundamentação e embasamento teórico para a concepção do modelo de processo de design de interação para DDS. Com o modelo concebido e a sua aplicação em um ou mais projetos de software no contexto distribuído, acredita-se na geração de conhecimento decorrente da concepção e aplicação do modelo.

Os resultados parciais sugerem a necessidade de mais pesquisas envolvendo design de interação e DDS, pois poucos estudos investigaram como solucionar problemas e desafios de design de interação em DDS.

Dado que o objetivo da pesquisa é conceber um modelo de processo de design de interação para DDS, espera-se que esse modelo seja capaz de apoiar o projeto e desenvolvimento de software no contexto distribuído que atenda às expectativas e objetivos dos usuários e contribua na participação de usuários finais e designers em seu desenvolvimento.

Espera-se contribuir na consolidação das ações e pesquisas nas áreas de Engenharia de Software e IHC, incentivando a adoção e a construção participativa de software.

Espera-se também que este estudo proporcione à comunidade científica um modelo baseado em evidências que possa contribuir para a condução de novas pesquisas e na concepção/adaptação de soluções de design de interação em DDS.

Ao final, espera-se:

- oferecer à comunidade científica evidências sobre problemas, desafios e soluções de design de interação em DDS;

- fornecer uma visão geral dos estudos que abordam design de interação em DDS e tendências de pesquisas;

- conceber e avaliar um modelo de processo de design de interação para DDS;

- mostrar a importância da integração de processos e soluções de design de interação em DDS.

\section{CONSIDERAÇÕES PARCIAIS}

Neste artigo, apresentou-se um projeto de pesquisa de doutorado em desenvolvimento que visa conceber um modelo de processo de design de interação para DDS. Resultados parciais sugerem a necessidade de mais pesquisas envolvendo design de interação e DDS. Pesquisas científicas em design de interação podem contribuir para a solução de problemas e desafios relacionados à interação humano-computador em DDS e, assim, melhorar a qualidade do produto desenvolvido.

Enfatiza-se a relevância do tema e destaca-se a importância de design de interação em DDS. Acredita-se que os problemas e desafios de design de interação ocasionado pela distribuição geográfica dos participantes diminui à medida que novas soluções de design de interação são desenvolvidas e problemas e desafios resolvidos.

\section{AGRADECIMENTOS}

Agradecemos o apoio financeiro da Coordenação de Aperfeiçoamento de Pessoal de Nível Superior (CAPES) e Instituto Federal de Mato Grosso (IFMT) por meio do Programa de Formação Doutoral Docente - Prodoutoral. Agradecemos também ao Grupo de Pesquisa e Extensão em Informática, Educação e Sociedade Onda Digital/UFBA. 


\section{REFERÊNCIAS}

1. D. D. Alves, M. I. Cagnin, and D. M. B. Paiva. 2014. Accessibility in development of free software projects. In 2014 XL Latin American Computing Conference (CLEI).

2. D. D. Alves and E. M. de Souza. 2017. Interaction Design in Free/Libre/Open Source Software Development: A Systematic Mapping. In Proceedings of the XVI Brazilian Symposium on Human Factors in Computing Systems (IHC 2017). ACM, New York, NY, USA.

3. J. L. N. Audy and R. Prikladnicki. 2008. Desenvolvimento distribuído de software. Elsevier.

4. P. M. Bach and J. M. Carroll. 2010. Characterizing the dynamics of open user experience design: The cases of Firefox and OpenOffice. org. Journal of the Association for Information Systems (2010).

5. P. M. Bach and M. Twidale. 2010. Involving Reflective Users in Design. In Proceedings of the SIGCHI Conference on Human Factors in Computing Systems (CHI'10). ACM, New York, NY, USA.

6. E. Carmel. 1999. Global Software Teams: Collaborating Across Borders and Time Zones. Prentice Hall PTR, Upper Saddle River, NJ, USA.

7. G. Çetin and M. Göktürk. 2008. A measurement based framework for assessment of usability-centricness of open source software projects. In Signal Image Technology and Internet Based Systems, 2008. SITIS'08. IEEE International Conference on. IEEE.

8. K. Crowston, K. Wei, J. Howison, and A. Wiggins. 2008. Free/Libre Open-source Software Development: What We Know and What We Do Not Know. ACM Comput. Surv. 44, 2 (March 2008).

9. A. Dresch, D. P. Lacerda, and J. A. V. Antunes Jr. 2015. Design science research: método de pesquisa para avanço da ciência e tecnologia. Bookman Editora.

10. GitHub. 2017. The State of the Octoverse 2017. (2017). Acessado em: 04 abr. 2018. Disponível em: https://octoverse.github.com/.

11. Dorina C Gumm, Monique Janneck, and Matthias Finck. 2006. Distributed participatory design-a case study. In Proceedings of the DPD Workshop at NordiCHI, Vol. 2.

12. J. D. Herbsleb. 2007. Global Software Engineering: The Future of Socio-technical Coordination. In 2007 Future of Software Engineering (FOSE '07). IEEE Computer Society, Washington, DC, USA.

13. A. R. Hevner, S. T. March, J. Park, and S. Ram. 2004. Design Science in Information Systems Research. MIS Q. (2004).

14. N. Iivari. 2011. Participatory Design in OSS Development: Interpretive Case Studies in Company and Community OSS Development Contexts. Behav. Inf. Technol. 30, 3 (May 2011).

15. M. Jiménez and M. Piattini. 2008. Problems and solutions in distributed software development: a systematic review. In International Conference on
Software Engineering Approaches for Offshore and Outsourced Development. Springer.

16. F. Lanubile, C. Ebert, R. Prikladnicki, and A. Vizcaíno. 2010. Collaboration tools for global software engineering. IEEE software 27, 2 (2010).

17. V. Lichtner, A. P. Kounkou, A. Dotan, J. P. Kooken, and N. A.M. Maiden. 2009. An Online Forum As a User Diary for Remote Workplace Evaluation of a Work-integrated Learning System. In CHI '09 Extended Abstracts on Human Factors in Computing Systems (CHI EA '09). ACM, New York, NY, USA.

18. A. Lisowska Masson, T. Amstutz, and D. Lalanne. 2017. A Usability Refactoring Process for Large-Scale Open Source Projects: The ILIAS Case Study. In Proceedings of the 2017 CHI Conference Extended Abstracts on Human Factors in Computing Systems (CHI EA '17). ACM, New York, NY, USA.

19. S. Luz and M. Masoodian. 2014. Involving Geographically Distributed Users in the Design of an Interactive System. In Proceedings of the Fifteenth Australasian User Interface Conference (AUIC '14).

20. J. Preece, H. Sharp, and Y. Rogers. 2015. Interaction design: beyond human-computer interaction (4 ed.). Wiley.

21. N. P. Radtke, M. A. Janssen, and J. S. Collofello. 2009. What makes Free/Libre Open Source Software (FLOSS) projects successful? An agent-based model of FLOSS projects. International Journal of Open Source Software and Processes (IJOSSP) 1, 2 (2009).

22. E. Raymond. 1999. The cathedral and the bazaar. Philosophy \& Technology 12, 3 (1999).

23. C. R. Reis and R. P. de M. Fortes. 2003. Caracterização de um Processo de Software para Projetos de Software Livre. Ph.D. Dissertation. PhD thesis, University of São Paulo, Brazil.

24. L. Santaella and J. A. Vieira. 2008. Metaciência como guia da pesquisa: uma proposta semiótica e sistêmica. São Paulo: Mérito (2008).

25. SourceForge. 2018. Make Your Projects Come To Life. (2018). Acessado em: 04 abr. 2018. Disponível em: https://sourceforge.net/.

26. R. Subramanyam and M. Xia. 2008. Free/Libre Open Source Software development in developing and developed countries: A conceptual framework with an exploratory study. Decision support systems 46, 1 (2008).

27. J. Treviranus. 2009. You say tomato, I say tomato, let's not call the whole thing off: the challenge of user experience design in distributed learning environments. On the Horizon 17 (2009).

28. G. Walsh and E. Foss. 2015. A Case for Intergenerational Distributed Co-design: The Online Kidsteam Example. In Proceedings of the 14th International Conference on Interaction Design and Children (IDC '15). ACM, New York, NY, USA. 